\title{
Special Issue: Direct and Large Eddy Simulation
}

\author{
Maria Vittoria Salvetti ${ }^{1} \cdot$ Jochen Fröhlich $^{2} \cdot$ Bernard J. Geurts $^{3} \cdot$ Hans Kuerten $^{4}$ \\ Published online: 4 August 2018 \\ (C) Springer Nature B.V. 2018
}

\section{Preface}

This Special Issue contains extended versions of 16 papers, selected from a total of about 120 contributions presented at the ERCOFTAC workshop Direct and Large-Eddy Simulation (DLES11), held in Pisa, Italy on May 29-31, 2017. The DLES Workshop series, which started in 1994, focuses on modern techniques designed to simulate turbulent flows based on a partial or full resolution of the instantaneous turbulent flow structures, such as Direct Numerical Simulation (DNS), Large-Eddy Simulation (LES) and hybrid models combining LES and RANS approaches.

With the growing capacity of modern computers and alongside significant progress in computational techniques as well as in closure modelling, turbulence-resolving approaches are nowadays applied to ever more complex flow problems and configurations, both in academic research and industrial practice, and they will undoubtedly be further enhanced and more widely applied in the future. The increasing complexity of the simulated problems and the use of turbulence-resolving approaches in an engineering context bring new challenges and pose open questions. For example, numerical methods are needed that are highly accurate, but are also able to deal with complex geometries and/or with physical phenomena interacting with turbulence-e.g., combustion and heat transfer. Furthermore,

Maria Vittoria Salvetti

mv.salvetti@ing.unipi.it

Jochen Fröhlich

Jochen.Froehlich@tu-dresden.de

Bernard J. Geurts

b.j.geurts@utwente.nl

Hans Kuerten

J.G.M.Kuerten@tue.nl

1 University of Pisa, Pisa, Italy

2 TU Dresden, Dresden, Germany

3 University of Twente, Enschede, The Netherlands

4 TU Eindhoven, Eindhoven, The Netherlands 
for LES and hybrid RANS/LES simulations, physical models must be developed, improved and validated for an increasing complexity and variety of applications. On the other hand, the increasing availability of DNS data sets provides a detailed and accurate reference for validating other approaches to turbulence and for guiding the development of physical models. DLES11 offered an opportunity to survey and discuss recent advances on all the above fronts and in several different application areas.

The Special Issue aims at providing an archival record of some of the best papers presented at DLES11. They cover methodological aspects, i.e. advances in numerical methods, SGS modelling, boundary conditions, post-processing and data analysis, and applications in several fields - namely, multiphase and reactive flows, dense gases, convection and heat transfer, aerodynamics of aerofoils and wings, bluff-body and separated flows, and transition. In this respect, the present Special Issue is closely consonant with the aims and ethos of FTaC. The selected papers were subjected to the same rigorous review process as that followed for any other contribution to FTaC.

The Special Issue Guest Editors

Maria Vittoria Salvetti

Jochen Fröhlich

Bernard J. Geurts

Hans Kuerten

Publisher's Note Springer Nature remains neutral with regard to jurisdictional claims in published maps and institutional affiliations. 